\title{
Tunable Plexcitonic Nanoparticles: A Model System for Studying Plasmon-Exciton Interaction from the Weak to the Ultrastrong Coupling Regime
}

\author{
Sinan Balci, ${ }^{* \dagger}$ Betul Kucukoz, ${ }^{\ddagger}$ Osman Balci, ${ }^{\S}$ Ahmet Karatay, ${ }^{\ddagger}$ Coskun Kocabas, ${ }^{\S}$ and Gul Yaglioglu*, ${ }^{*}$ \\ ${ }^{\dagger}$ Department of Astronautical Engineering, University of Turkish Aeronautical Association, 06790 Ankara, Turkey \\ ${ }^{\ddagger}$ Department of Engineering Physics, Faculty of Engineering, Ankara University, Besevler, 06100 Ankara, Turkey \\ ${ }^{\S}$ Department of Physics, Bilkent University, 06800 Ankara, Turkey
}

\begin{abstract}
Controlling the number of dye molecules on metallic nanoparticles, which in turn affects the magnitude of Rabi splitting energy, is crucial for obtaining hybrid metal core-organic shell nanoparticles with tunable optical properties in the visible spectrum since the magnitude of the Rabi splitting energy directly determines the strength of the coupling between plasmonic nanoparticles and dye molecules. In this work, we present a new method for the synthesis of plexcitonic nanoparticles, and thus we are able to control the number of dye molecules self-assembled on $\mathrm{Ag}$ nanoprisms ( $\mathrm{Ag} \mathrm{NPs}$ ) by adjusting the concentration of dye molecules used in the synthesis. Indeed, individual dye molecules

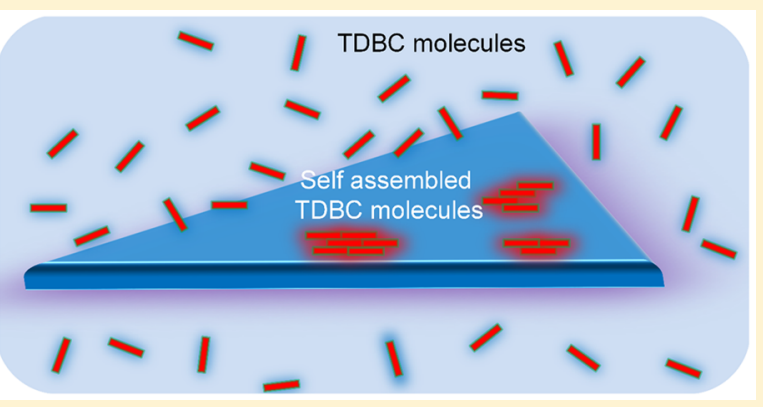
self-assemble into J-aggregates on Ag NPs. Thus, in the finite-element simulations and experimental data of the hybrid metal organic nanoparticles, we observed a transition from weak coupling to the ultrastrong coupling regime. Besides, ultrafast energy transfer between plasmonic nanoparticles and excitonic aggregated dye molecules has been extensively studied as a function of Rabi splitting energy. We observe that the lifetime of the polariton states increases with the coupling strength and the upper polaritons are short-lived, whereas the lower polaritons are long-lived. Hybrid metal-organic nanoparticles presented in this study (i) have tunable Rabi splitting energies, (ii) are easy to prepare in large quantities in aqueous medium, (iii) can be uniformly assembled on solid substrates, (iv) have resonance frequencies in the visible spectrum, and (v) have small mode volume, thus making them an excellent model system for studying light-matter interaction at nanoscale dimensions from the weak to ultrastrong coupling regime.
\end{abstract}

KEYWORDS: plexcitons, plasmons, J-aggregates, excitons, nanoprisms, strong coupling, Rabi splitting

$\mathrm{E}$ ngineering and controlling light-matter interaction at the nanoscale dimension ${ }^{1}$ has attracted a surge of interest in recent years owing to the direct impact of the studies on lightemitting devices, solar cells, plasmonic circuits, biosensors, nanoscale light sources, energy transport, quantum optics, etc. $^{2-7}$ Specifically, formation of hybrid nanostructures between metallic nanoparticles supporting localized surface plasmon polaritons (LSPPs) and quantum emitters supporting excitons, for example, J-aggregates, organic dyes, and quantum dots, can lead to the development of novel nanoscale devices with greatly improved performances and the emergence of new optical hybrid states such as plexcitons ${ }^{8}$ and plexcimons. ${ }^{9,10}$

When metal thin films, metal gratings, and metal nanoparticles are uniformly covered with J-aggregates, dye molecules, and quantum dots, a new hybridized optical state called a plexciton emerges in the strong coupling regime. ${ }^{11}$ High quality factor plasmonic nanostructures and dye molecules with large oscillator strengths are needed in order to reach the strong coupling regime. Depending on the quality factor of the plasmon resonance in plasmonic systems and the oscillator strength and concentration of the quantum emitter, different coupling regimes have been realized. ${ }^{11}$ In the so-called weak coupling regime (WCR), the original optical modes of either the plasmonic or excitonic system have not been modified, whereas emission or absorption of the nanoparticles or molecules is greatly enhanced, i.e., the Purcell effect. ${ }^{12,13}$ However, in the strong coupling regime (SCR), the new hybrid system shows new optical modes whose frequencies are completely different from the original optical modes, i.e., bare plasmon and exciton modes. ${ }^{14}$ Indeed, the coupling strength overcomes the dissipative losses in the strongly coupled system. ${ }^{15}$ If the positions of new optical modes are plotted as a function of detuning, a very clear anticrossing behavior is observed with a minimum energy separation at zero detuning called the Rabi splitting energy, which determines the strength of the coupling between exciton and plasmon. ${ }^{16}$ Now, the coupling is coherent and the plasmon-exciton interaction becomes reversible; that is, the plasmon and exciton become entangled, and hence new physics can be observed such as

Received: July 14, 2016

Published: October 31, 2016 
thresholdless lasing and stimulated emission. ${ }^{17}$ When the magnitude of the Rabi splitting energy becomes comparable to the bare quantum emitter resonance energy, the light-matter interaction reaches a new regime of ultrastrong coupling (UCR). ${ }^{18}$

Up until now, in order to synthesize plexcitonic nanoparticles, metallic shells, ${ }^{8,19}$ gold nanowires, ${ }^{19-22}$ nanoprisms, ${ }^{10,23}$ spherical nanoparticles, ${ }^{19,24,25}$ and recently nanodisks $^{26}$ have been used as a plasmonic component. On the other hand, J-aggregates (JAs), aggregated dye molecules with a very narrow absorption band that is red-shifted to a longer wavelength with respect to the monomer absorption band, have been mostly used as an excitonic component in the plexcitonic nanoparticle synthesis due to their very narrow absorption band and very large oscillator strength. ${ }^{27}$ Nevertheless, in the previous studies, the Rabi splitting energy of the plexcitonic nanoparticles is not tunable ${ }^{10}$ and thus their optical properties cannot be tailored, which limits their practical and fundamental applications such as ultrafast energy transfer between plasmon and exciton as a function of coupling strength and controlling group velocity of plexcimons. ${ }^{9}$ Here, for the first time, we were able to synthesize plexcitonic nanoparticles with tunable Rabi splitting energies in an aqueous medium. Therefore, in the hybrid metal core-organic shell nanoparticles a transition from weak coupling to ultrastrong coupling regime was observed. Owing to the fact that the nanoparticles (i) have tunable Rabi splitting energies, (ii) have resonance frequencies in the visible, (iii) are easy to prepare in aqueous medium and can be assembled on solid substrates, and (iv) have small mode volume $\left(\sim 10^{4} \mathrm{~nm}^{3}\right)$, they will find new applications in a variety of light-matter studies such as quantum optics, nanoscale energy transfer, and nanoscale light sources as well. ${ }^{9,28}$ In this study, ultrafast energy transfer between plasmons and excitons $^{29}$ has been extensively studied as a function of the coupling strength.

$\mathrm{Ag}$ nanoprisms (Ag NPs) of controllable edge length, displaying remarkable optical properties in the visible and near-infrared spectrum, were synthesized by a seed-mediated protocol in aqueous medium at room temperature. ${ }^{9,10}$ In this work, Ag NPs were used as the plasmonic nanostructure since they have broadly tunable localized plasmon resonance wavelengths ranging from 400 to $1100 \mathrm{~nm} .^{30}$ Spherical Ag nanoparticles were used as seed nanoparticles. ${ }^{31}$ Millipore water was used throughout all experiments, and all reactions were performed in aqueous medium at room temperature. The seed nanoparticles were synthesized by combining $5 \mathrm{~mL}$ of 2.5 $\mathrm{mM}$ trisodium citrate solution with $0.25 \mathrm{~mL}$ of $500 \mathrm{mg} / \mathrm{L}$ poly(sodium 4-styrenesulfonate) (PSS) and $0.3 \mathrm{~mL}$ of freshly prepared $10 \mathrm{mM} \mathrm{NaBH}_{4}$. Subsequently, $5 \mathrm{~mL}$ of $0.5 \mathrm{mM}$ $\mathrm{AgNO}_{3}$ was added drop by drop at a rate of $\sim 2 \mathrm{~mL} / \mathrm{min}$ to this solution combination under stirring. In less than $30 \mathrm{~min}$, the color of the solution turned deep yellow, which is an indication of Ag nanoparticle formation. The spherical Ag nanoparticles have an LSPP resonance wavelength at $\sim 400 \mathrm{~nm}$. Ag NPs were synthesized by varying the amount of seed nanoparticles added to the reaction solution. ${ }^{10}$ The number of seed nanoparticles in the reaction medium directly controls the morphology (edge length) and hence the LSPP resonance wavelength of the Ag NPs. For instance, in order to synthesize Ag NPs at $\sim 750 \mathrm{~nm}$ LSPP resonance wavelength, $5 \mathrm{~mL}$ of Millipore water was combined with $75 \mu \mathrm{L}$ of $10 \mathrm{mM}$ ascorbic acid and $60 \mu \mathrm{L}$ of the seed solution. Actually, by tuning the amount of spherical seed nanoparticles in the reaction medium, the LSPP resonance wavelength of the Ag nanoprisms can be varied from the visible part of the electromagnetic spectrum $(400 \mathrm{~nm})$ to the nearinfrared part of the electromagnetic spectrum $(1100 \mathrm{~nm})$. Ultimately, the synthesized $\mathrm{Ag}$ nanoprisms were stabilized by adding $0.5 \mathrm{~mL}$ of $25 \mathrm{mM}$ trisodium citrate aqueous solution.

A cyanine dye, (5,5',6,6'-tetrachlorodi(4-sulfobutyl)benzimidazolocarbocyanine (TDBC), purchased from FEW Chemicals, was used without further purification. Two different methods, Br-assisted self-assembly of dye molecules at high dye concentration as reported earlier ${ }^{9,10}$ and, in this study, selfassembly of dye molecules at low concentration, were utilized to chemically synthesize plexcitonic nanoparticles. In the $\mathrm{Br}$ assisted synthesis process, ${ }^{9,10}$ a $1 \mathrm{mM}$ TDBC aqueous solution was prepared in $1 \mathrm{mM} \mathrm{KBr}$, and then $\mathrm{Ag} \mathrm{NPs}$ in water were combined with the TDBC solution in $\mathrm{KBr}$. Fast color variation in the solution indicates plexcitonic nanoparticle formation. Excess cyanine dye molecules in the solution were removed by centrifugation at $15000 \mathrm{rpm}$ for $10 \mathrm{~min}$, and the pellet was suspended in water. It should be mentioned that, in the $\mathrm{Br}$ assisted synthesis process, the number of dye molecules on the Ag NPs cannot be manipulated because, at low dye concentration, $\mathrm{Ag}$ NPs degrade in the presence of $\mathrm{Br}$ ions in the medium. ${ }^{10}$ In the second process, a low concentration of TDBC molecules (smaller than $0.1 \mathrm{mM}$ ) in Br-free solution was directly added to the Ag NP solution in order to selfassemble TDBC molecules on Ag NPs. Noteworthily, adsorption of dye molecules to various nanoparticles and surfaces has been extensively studied and known for many years mainly because of the understanding of the enhancement effects observed in surface-enhanced Raman spectroscopy spectra. ${ }^{31,32}$ In a typical experiment, varying amounts of 0.1 $\mathrm{mM}$ TDBC in water $(25,50,100,200,300$, and $400 \mu \mathrm{L})$ were added to $1 \mathrm{~mL}$ of $\mathrm{Ag} \mathrm{NP}$ solution. The strength of the plasmon-exciton coupling determined by the magnitude of the Rabi splitting energy was controlled with the amount of dye molecules in the medium. Besides that, the concentration of the starting TDBC solution was further decreased to $0.01 \mathrm{mM}$. Varying amounts of $0.01 \mathrm{mM}$ TDBC were added to $1 \mathrm{~mL}$ of $\mathrm{Ag}$ $\mathrm{NP}$ solution. At such a low concentration of dye molecules $\left(\sim 10^{-6} \mathrm{M}\right)$ no J-aggregation of TDBC molecules is expected, ${ }^{27}$ and accordingly individual TDBC molecules self-assemble and directly form J-aggregates on the Ag NP surface.

Transient absorption measurements were performed with a femtosecond pump-probe method utilizing a Ti:sapphire laser amplifier-optical parametric amplifier system (Spectra Physics) with a $50 \mathrm{fs}$ pulse duration and $1 \mathrm{kHz}$ repetition rate. ${ }^{33} \mathrm{~A}$ commercial pump-probe experimental setup with a white light continuum probe beam (Spectra Physics) was used. Experiments were performed in transmission geometry at $40 \mu \mathrm{J} / \mathrm{cm}^{2}$ pump fluencies with $100 \mathrm{fs}$ pulse duration. A positive sign in the pump probe data $\Delta T / T$ corresponds to decreased transmission (excited-state absorption (ESA)), whereas a negative sign of $\Delta T / T$ corresponds to increased transmission (bleach signal), according to our sign convention. The linear absorption spectra of dye molecules and plasmonic and plexcitonic nanoparticles were obtained using an Agilent Technologies Cary Series UVvis spectrophotometer.

A schematic representation of the self-assembly of individual TDBC dye molecules on Ag NPs is depicted in Figure 1a. Extinction spectra of bare Ag NPs as well as $0.1 \mathrm{mM}$ TDBC dyes are provided in Figure $1 \mathrm{~b}$. The broad absorbance band at around $\sim 513 \mathrm{~nm}$ in the dye spectrum is an indication of the presence of monomers or individual TDBC dye molecules in 


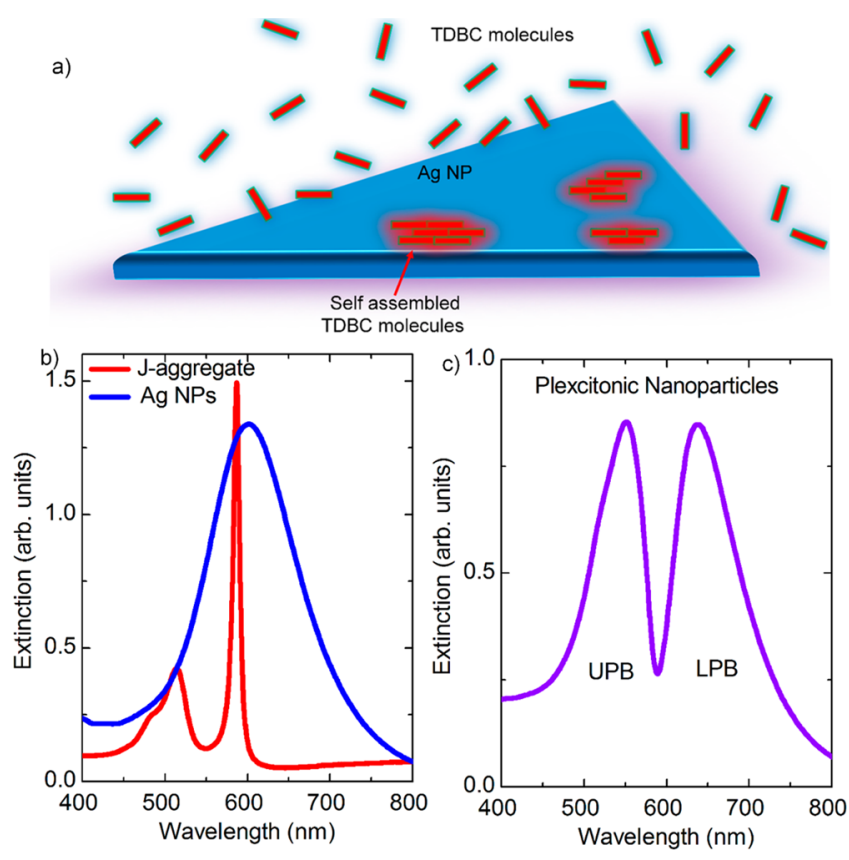

Figure 1. Plexcitonic nanoparticle formation: Self-assembly of dye molecules on Ag NPs. (a) Schematic representation of the selfassembly process. (b) Extinction spectra of Ag NPs and $0.1 \mathrm{mM}$ TDBC in aqueous solution. Localized plasmon resonance of the $\mathrm{Ag}$ NPs is at around $600 \mathrm{~nm}$. The broad absorbance peak at around $~ 513$ $\mathrm{nm}$ is a TDBC monomer spectrum, and the sharp absorbance peak at around $\sim 587 \mathrm{~nm}$ originates from the aggregated TDBC molecules. (c) Extinction spectrum of plexcitonic nanoparticles formed by selfassembly of individual TDBC molecules on Ag NPs in aqueous solution. Two new polariton branches are formed.

the solution. However, a very sharp absorbance band at around $587 \mathrm{~nm}$ is a very strong indication of J-aggregate formation of TDBC molecules. It should be noted here that pump-probe experiments have demonstrated that the delocalization length of the electronically excited state, i.e., Frenkel excitons in TDBC J-aggregates, is $\sim 15$ TDBC molecule lengths. ${ }^{34}$ It is well known that cyanine dyes form J-aggregates at high concentration $\left(>\sim 10^{-6} \mathrm{M}\right)$ in basic medium $(\mathrm{pH} \sim 11) .{ }^{27,34}$ Plexcitonic nanoparticles are formed when Ag NPs are combined with a TDBC dye solution. Consequently, two new hybrid bands, namely, an upper polariton branch (UPB) and a lower polariton branch (LPB), are formed, Figure 1c. In fact, the energy separation between the new polariton branches gives the value of the Rabi splitting energy determining the strength of the coupling between plasmon and exciton. Noteworthily, it has been theoretically predicted that when $n$ resonances (two resonances, i.e., plasmon and exciton resonances) are simultaneously excited in the coupled system $n-1$ (one resonance, i.e., the $\operatorname{dip}$ at $\sim 587 \mathrm{~nm}$ ), transparency dips arise in the dispersion curve. ${ }^{35}$

Plexcitonic nanoparticles with easily tunable Rabi splitting energies are desperately needed to study light-matter interaction in a broad range of Rabi splitting energy values. We here report the synthesis of plexcitonic nanoparticles with different Rabi splitting energies, Figure $2 \mathrm{a}$. The volume of 0.1 $\mathrm{mM}$ TDBC dye added to the $1000 \mu \mathrm{L}$ of Ag NPs was increased from sample 1 to 6 . For example, in sample 2, $100 \mu \mathrm{L}$ of 0.1 $\mathrm{mM}$ dye solution was added to $1000 \mu \mathrm{L}$ of $\mathrm{Ag} \mathrm{NP}$ solution, and no Rabi splitting was observed in the extinction spectrum; hence the nanoparticles are in the weak coupling regime.
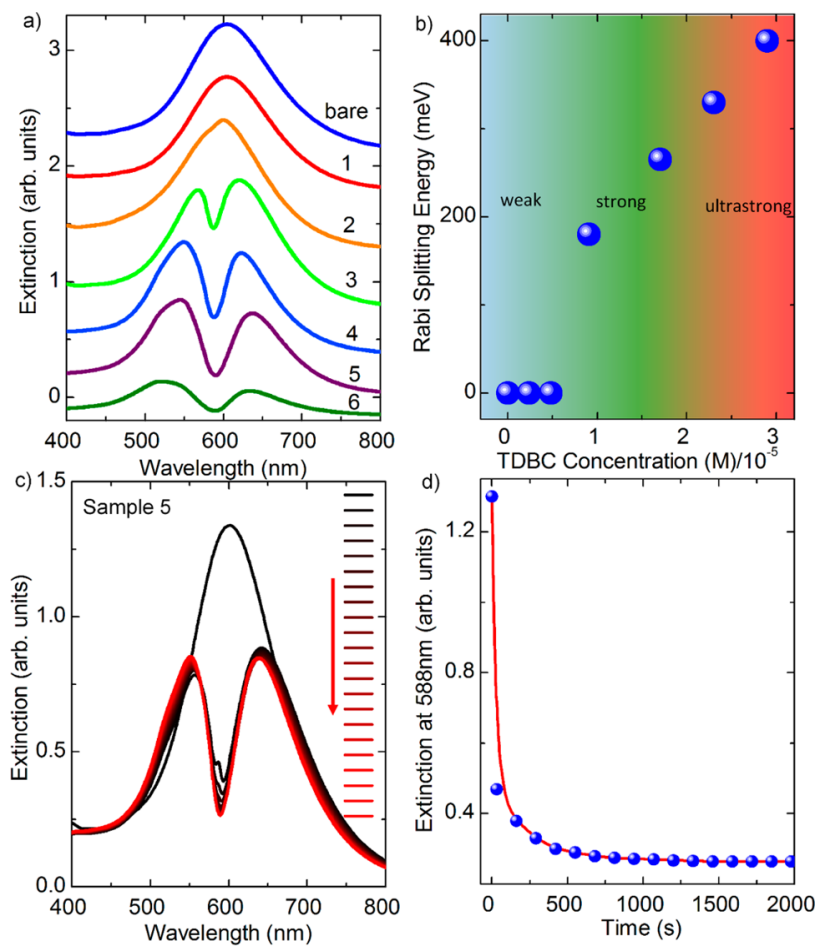

Figure 2. Tunable plexcitonic nanoparticles. (a) Extinction spectra of Ag NPs synthesized with varying concentrations of TDBC molecules. The amount of TDBC molecules in the Ag NPs solution increases from sample 1 to 6 . The TDBC concentration is $0.1 \mathrm{mM}$, and the volume of the dye varies. (b) Rabi splitting energy increases with the concentration of TDBC. As the concentration of dye molecules increases, a transition from weakly coupling to ultrastrong coupling regime is observed. (c) Extinction spectra of sample 4 as a function of time; each spectrum is taken after $\sim 2$ min. (d) Plot of extinction at $587 \mathrm{~nm}$ vs time for sample 4 . As clearly shown in the figure, in less than a few minutes the plexcitonic nanoparticles are directly formed.

Indeed, at least $\sim 15$ TDBC molecules are needed to form a single J-aggregate unit. ${ }^{34}$ In fact, in a classical description of coupled oscillators, the Rabi splitting energy increases with the number of particles in the medium:

$$
\Omega=\omega_{+}-\omega_{-}=\sqrt{\frac{N}{V} \frac{e^{2}}{\varepsilon_{0} m}}
$$

where $\Omega, N, V, \omega_{+}, \omega_{-}, e, m$, and $\varepsilon_{0}$ are Rabi splitting frequency, number of particles, volume, UPB frequency, LPB frequency, electron charge, electron mass, and permittivity of free space, respectively. Depending on the dye concentration, the coupling can be divided into three main parts: weak coupling regime (no splitting in the bare optical modes), strong coupling regime (new optical modes emerge as UPB and LPB), and finally ultrastrong coupling regime (Rabi splitting energy is at least $\sim 15 \%$ of the bare plasmon or exciton resonance energy ${ }^{18}$ ), Figure $2 \mathrm{~b}$. It should be noted here that in a previous study the number of dye molecules on the plexcitonic nanoparticles was irreversibly controlled by laser-induced photodegradation. ${ }^{28}$ In another study, the Rabi splitting energy was reversibly switched by using photochromic dye molecules in a large cavity (not a plexcitonic nanoparticle) formed by two flat silver thin films of $35 \mathrm{~nm}$ thickness. ${ }^{18}$ In addition, we studied the kinetics of Jaggregate formation on the surface of $\mathrm{Ag}$ NPs and hence plexcitonic nanoparticle formation in aqueous medium as shown in Figure $2 \mathrm{c}$,d. The rate of plexciton formation was 

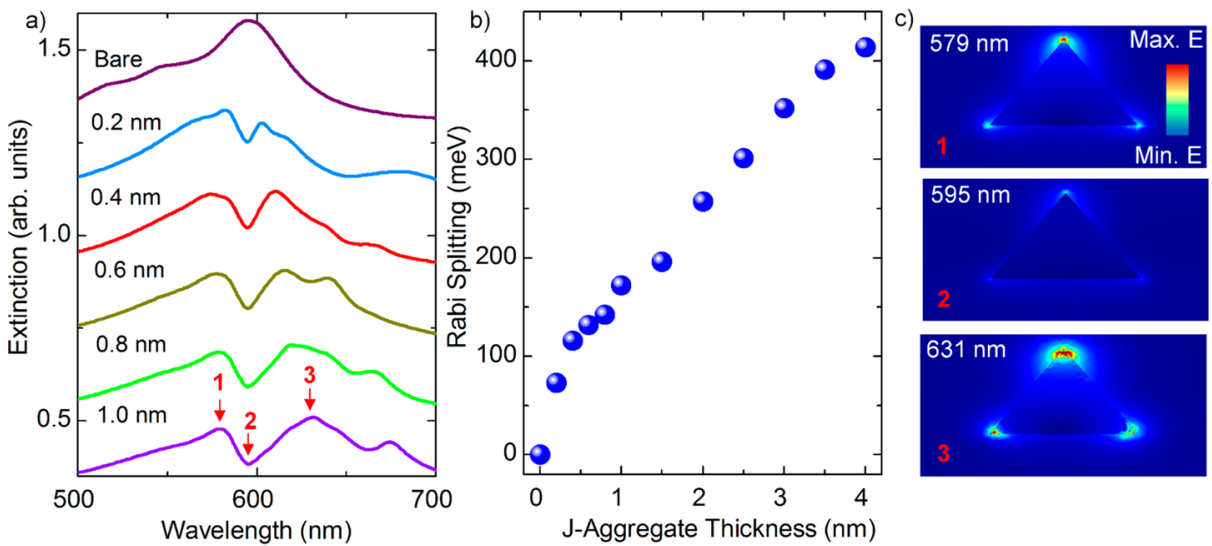

Figure 3. Electrodynamics simulations of tunable plexcitonic nanoparticles. (a) Extinction spectra of a single Ag NP with varying J-aggregate film thickness computed using COMSOL. The J-aggregate film thickness ranges from 0.2 to $1.0 \mathrm{~nm}$. The Rabi splitting energy, the separation between the polaritonic branches, increases with the dye film thickness. (b) Calculated Rabi splitting energy values from the extinction spectra in (a). Notably, the Rabi splitting energy increases with the J-aggregate film thickness, which is in close agreement with the experimental results shown in Figure 2. (c) Distribution of the calculated electric field on the single plexcitonic nanoparticle for three different incident wavelengths: (1) $579 \mathrm{~nm}$, (2) 595 $\mathrm{nm}$, and (3) $635 \mathrm{~nm}$.

evaluated from the variation of absorbance at the extinction resonance of $587 \mathrm{~nm}$. Indeed, the plexcitonic nanoparticle formation is relatively fast and occurs within a few minutes. Previously, the kinetics of J-aggregate formation (showing an absorbance maximum at around $409 \mathrm{~nm}$ ) on spherical gold nanoparticle colloids having a localized plasmon resonance wavelength at around $550 \mathrm{~nm}$ were vastly studied, and the Jaggregate formation rate was measured to be less than a minute. $^{36}$ This value is similar to the values reported here; however direct comparison is again difficult since (i) the nanoparticles and dyes used in the previous study are different from the nanoparticles and dyes used in this work and (ii) Jaggregate absorption resonance frequency and gold nanoparticle plasmon resonance frequency are far away from each other so that there is no plexcitonic nanoparticle formation. ${ }^{36}$ Furthermore, electrodynamic simulations of single plexcitonic nanoparticles were done using the commercially available finite element method COMSOL, Figure 3a. A single Ag nanoprism with an edge length of $54 \mathrm{~nm}$ and a thickness of $10 \mathrm{~nm}$ was suspended in air. Optical constants of the J-aggregate film were taken from the previous studies. ${ }^{16,37}$ The Rabi splitting energy increases with the J-aggregate film thickness. The agreement between the simulation and experimental data is generally very good. For example, the calculated Rabi splitting energy (i.e., $\sim 400 \mathrm{meV}$ ) of the plexcitonic nanoparticle with a $4 \mathrm{~nm}$ thick Jaggregate film matches the Rabi splitting energy of the plexcitonic nanoparticles synthesized by using a $3 \times 10^{-5} \mathrm{M}$ dye solution, Figure $3 b$.

The plexcitonic nanoparticles shown in Figure 2 were synthesized by using a $0.1 \mathrm{mM}$ TDBC solution where most of the dye molecules are in aggregated form as shown in Figure 1b. Therefore, it can safely be concluded that the J-aggregates on Ag NPs are due to the assembly of (i) monomers or (ii) aggregated dye molecules. When TDBC concentration is lowered, for example $1 \times 10^{-5} \mathrm{M}$, the absorption spectrum shows a broad absorbance band at around $\sim 513 \mathrm{~nm}$, which demonstrates that all TDBC molecules are in the monomer form, as shown in Figure 4a. ${ }^{27}$ Now it is clear that there are not any aggregated dye molecules in the medium. Surprisingly, when a monomer dye solution is used in the synthesis, individual dye molecules self-assemble on Ag NPs and form
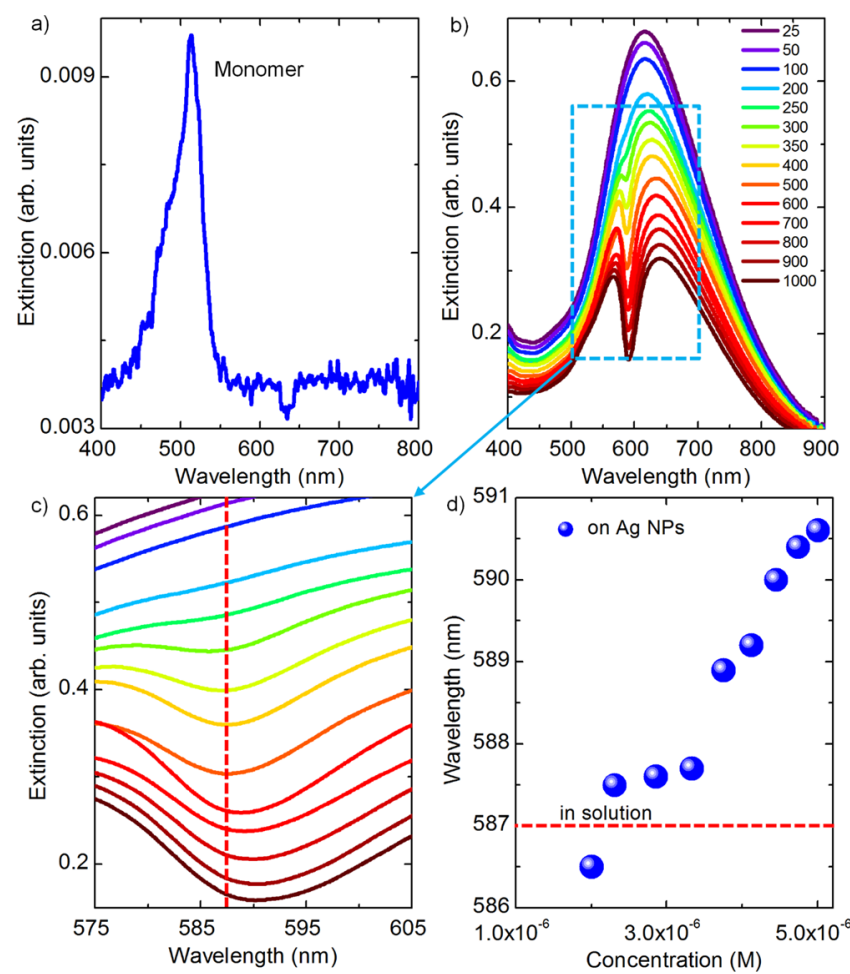

Figure 4. Plexcitonic nanoparticle formation with very low TDBC concentration, $1 \times 10^{-5} \mathrm{M}$. (a) Absorbance of a $1 \times 10^{-5} \mathrm{M}$ TDBC solution. The broad absorbance resonance at around $513 \mathrm{~nm}$ indicates that all TDBC molecules are in the monomer form. (b) Extinction spectra of the plexcitonic nanoparticles synthesized by adding varying volumes of $1 \times 10^{-5} \mathrm{M}$ TDBC solution in microliters. Rabi splitting energy increases as the volume of dye added to the Ag NP solution is increased. It is apparent from the spectra that individual TDBC molecules aggregate on the Ag NPs and form plexcitonic nanoparticles. (c, d) The maximum absorption wavelength of aggregated TDBC molecules on Ag NPs is modified with respect to the optical absorption $(\sim 587 \mathrm{~nm})$ in Ag NP-free solution.

plexcitonic nanoparticles, as shown in Figure $4 \mathrm{~b}$. The Rabi splitting energy increases with the volume of the dye solution added to the silver nanoprism solution. Here we assumed that 

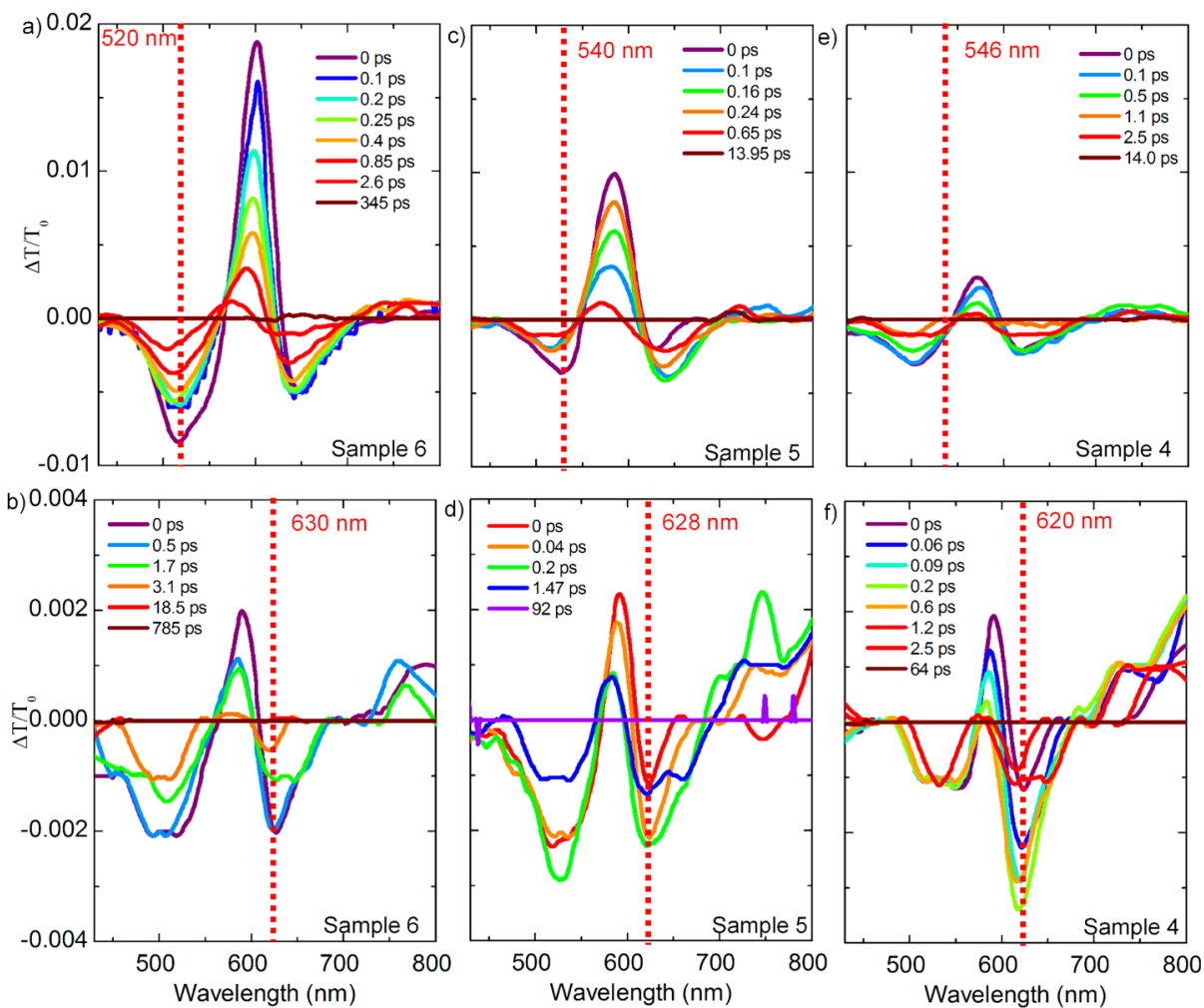

Figure 5. Ultrafast energy transfer between excitons and plasmons in tunable plexcitonic nanoparticles. Transient absorption spectra of plexcitonic nanoparticles,: (a, b) sample 6, (c, d) sample 5, and (e,f) sample 4 for varying time delays. The polaritonic branches are selectively excited for each sample, as indicated by a vertical dotted line corresponding to the central wavelength of the pump pulses. Linear absorption spectra of the samples are given in Figure 2a.

deep quenching exactly matches the absorption peak of the target molecule in the cell. Previously, the deep quenching in the plexcitonic nanoparticles extinction spectrum was used as a sensor to molecularly image biomolecules in living cells. ${ }^{38}$

A closer look at the spectra shown in Figure $4 \mathrm{~b}$ reveals that absorbance wavelength of the J-aggregate in solution, i.e., $\sim 587$ $\mathrm{nm}$, is progressively shifted toward longer wavelengths with an increase in the self-assembled dye molecules on the Ag NPs, Figure 4c,d. Recently, it has been clearly shown that absorbance of dye molecules on a metal nanoparticle surface is different from the absorbance of dye molecules in metal-nanoparticlefree solution owing to the modifications of the intrinsic dye polarizability upon adsorption on metallic nanoparticles. ${ }^{39}$ Therefore, our results support the conclusion obtained in the previous work. ${ }^{39}$

We now turn our attention to the ultrafast energy transfer between exciton and plasmon as a function of Rabi splitting energy. The transient absorption spectra of bare Ag NPs show a typical decreased absorption around $600 \mathrm{~nm}$ corresponding to a plasmon resonance band and increased absorption at the low energy side of the spectra corresponding to ESA as observed in our previous study. ${ }^{33}$ Transient absorption spectra of Ag NPs with dye molecules are totally different from the transient absorption spectra of the bare Ag NPs. The plasmon band has been quenched at the position of the exciton resonance energy value similar to the ground-state absorption spectra. In order to understand the effect of dye amount on the ultrafast dynamics of energy transfer, several Ag NPs-J-aggregate samples were prepared with varying amount of dye molecules, Figure 2 a. Transient absorption spectra of the samples 6, 5, 4, and 3 for various time delays pumped at the UPB and LPB are given in
Figure 5 and Figure 6. Transmission ultrafast pump-probe spectra show bleach signals for samples indicated in Figure 5 at around $500-550 \mathrm{~nm}$ along with a positive signal between two bleach signals regardless of the pump wavelengths. In our previous study, we obtained similar results for plexcitonic nanoparticles synthesized by using $\mathrm{Br}$-assisted synthesis. ${ }^{33}$ Bleach signals appear at zero time delay for both UPB and LPB of samples shown in Figure 5, which indicates that there is a coherent energy exchange between excitons and plasmons. ${ }^{29}$ The positive signal is assigned to ESA in the literature. ${ }^{40,41}$ The positive signal can be attributed to temporary electron transfer from J-aggregates to $\mathrm{Ag}$ NPs. In this sense, the coupling strength between plasmon and exciton increases from samples 3 to 6 , leading to evolution of the center positive signal. Our experimental setup time resolution is $100 \mathrm{fs}$, so that we could not observe electron transfer contribution to positive signal, which is faster than $50 \mathrm{fs}$. Another bleach signal appears at the LPB due to the strong coupling for samples demonstrated in Figure 5, as observed in our previous study. ${ }^{33}$ When the Rabi splitting energy (coupling strength) is very low, as it is for sample 3, transient absorption spectra look like bare plasmon spectra, Figure 6a,b.

The lifetime of the decay curves of the lower energy bleach signal $(630 \mathrm{~nm})$ is shorter for a UPB $(\sim 0,75 \mathrm{ps})$ than an LPB $(1,8 \mathrm{ps})$, which may be due to the nonradiative energy transfer from the UPB to lower energy states owing to the transition of upper branch polaritons to lower energy states through emission of phonons. ${ }^{42-44}$ Consequently, the following conclusions can be drawn from the pump-probe experiments: (i) Transient absorption spectra of the samples shift to the higher energy region due to shifting of the UPB and LPB for 

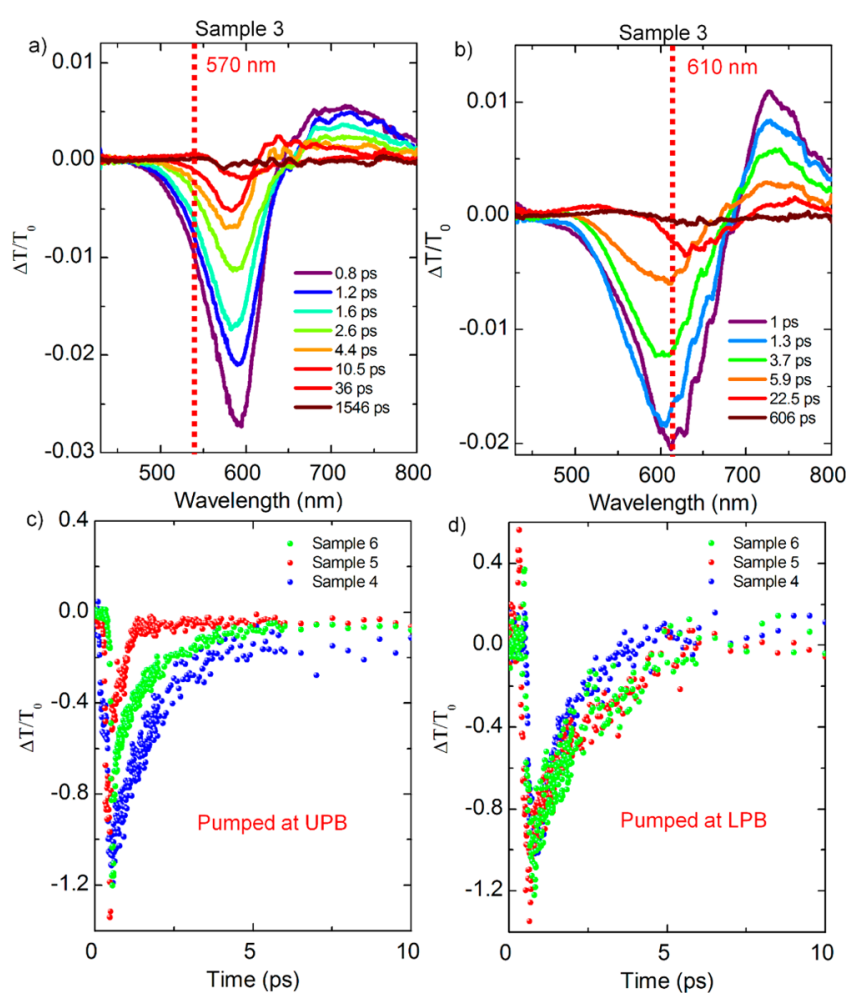

Figure 6. (a, b) Transient absorption spectra of sample 3 for varying time delays. UPB and LPB are selectivelly excited as indicated by a vertical dotted line corresponding to the central wavelength of the pump pulses. Sample 3 has the lowest Rabi splitting energy, which is less than $200 \mathrm{meV}$, among the samples studied here. A linear absorption spectrum of the same sample is given in Figure 2a. Decay curves of the bleach signals pumped at the (c) UPB and (d) LPB. As the coupling strength increases, the samples demonstrate a longer lifetime for both upper and lower bleach signals.

each sample. (ii) Sample 6, which has the largest Rabi splitting energy among the studied samples, exhibits the largest bleach and transient absorption signals for both pumping energies. (iii) As the coupling strength increases, the samples show longer lifetime for both upper and lower bleach signals due to electron oscillation between plasmon and exciton. Therefore, the longest lifetime was observed for sample 6, which has the largest Rabi splitting energy. (iv) In agreement with the previous studies, ${ }^{44}$ the lower polariton is long-lived but the upper polariton is short-lived.

In summary, we have demonstrated a simple, straightforward, but highly effective new route for controlling self-assembly of dye molecules on Ag nanoprisms. By simply adjusting the concentration of dye molecules in the reaction medium, plexcitonic nanoparticles with tunable optical properties in the visible spectrum have been synthesized. The synthesized nanoparticles show tunable Rabi splitting energies. A transition from the weak to the ultrastrong coupling regime has been observed. The finite element simulations and experimental data indicate that the Rabi splitting energy increases with the number of dye molecules assembled on the Ag nanoprisms. In addition, in this work, ultrafast energy transfer between plasmonic nanoparticles and excitonic aggregated dye molecules has been extensively studied as a function of Rabi splitting energy. Unlike the previous studies, we have shown that individual dye molecules self-assemble on Ag NPs and construct J-aggregate nanostructures right on the Ag nano- prisms. In less than a few minutes, the dye molecules directly and immediately self-assemble on Ag nanoprisms. The hybrid nanoparticles presented herein are easy to prepare in large quantities and can be assembled on a variety of solid substrates. The plexcitonic nanoparticles with tunable optical properties are very promising for a variety of applications including roomtemperature quantum optics, biosensors, nanoscale light sources, and nanoscale energy transport. ${ }^{7,45}$ Especially, controlling the number of dye molecules assembled on metal nanoprisms has a direct influence on the number of excitons ( $\sim 15$ dye molecules generate one exciton) coupled to plasmon polaritons on Ag NPs, and hence room-temperature-tunable quantum optics on a single Ag NP level can be envisaged. ${ }^{28}$ Another interesting application of the presented nanoparticles is controlling optical properties of plexcimons, which are a recently discovered plasmon-exciton-plasmon ultrahybrid optical state. ${ }^{9}$ For example, group velocity of plexcimons can be easily manipulated by controlling only the number of excitons on Ag NPs.

\section{AUTHOR INFORMATION}

\section{Corresponding Authors}

*E-mail: sbalci@thk.edu.tr.

*E-mail: Gul.Yaglioglu@eng.ankara.edu.tr.

\section{Notes}

The authors declare no competing financial interest.

\section{ACKNOWLEDGMENTS}

This work has been supported by a grant (112T091) from the TUBITAK.

\section{REFERENCES}

(1) Koenderink, A. F.; Alu, A.; Polman, A. Nanophotonics: Shrinking light-based technology. Science 2015, 348, 516-521.

(2) Hentschel, M.; Saliba, M.; Vogelgesang, R.; Giessen, H.; Alivisatos, A. P.; Liu, N. Transition from Isolated to Collective Modes in Plasmonic Oligomers. Nano Lett. 2010, 10, 2721-2726.

(3) Ow, H.; Larson, D. R.; Srivastava, M.; Baird, B. A.; Webb, W. W.; Wiesner, U. Bright and stable core-shell fluorescent silica nanoparticles. Nano Lett. 2005, 5, 113-117.

(4) Noginov, M. A.; Zhu, G.; Belgrave, A. M.; Bakker, R.; Shalaev, V. M.; Narimanov, E. E.; Stout, S.; Herz, E.; Suteewong, T.; Wiesner, U. Demonstration of a spaser-based nanolaser. Nature 2009, 460, 1110U68.

(5) Tame, M. S.; McEnery, K. R.; Ozdemir, S. K.; Lee, J.; Maier, S. A.; Kim, M. S. Quantum plasmonics. Nat. Phys. 2013, 9, 329-340.

(6) Manjavacas, A.; de Abajo, F. J. G.; Nordlander, P. Quantum Plexcitonics: Strongly Interacting Plasmons and Excitons. Nano Lett. 2011, 11, 2318-2323.

(7) Yuen-Zhou, J.; Saikin, S. K.; Zhu, T.; Onbasli, M. C.; Ross, C. A.; Bulovic, V.; Baldo, M. A.: Plexciton Dirac points and topological modes. Nat. Commun. 2016, 7.1178310.1038/ncomms11783

(8) Fofang, N. T.; Park, T. H.; Neumann, O.; Mirin, N. A.; Nordlander, P.; Halas, N. J. Plexcitonic Nanoparticles: PlasmonExciton Coupling in Nanoshell-J-Aggregate Complexes. Nano Lett. 2008, 8, 3481-3487.

(9) Balci, S.; Kocabas, C. Ultra hybrid plasmonics: strong coupling of plexcitons with plasmon polaritons. Opt. Lett. 2015, 40, 3424-3427.

(10) Balci, S. Ultrastrong plasmon-exciton coupling in metal nanoprisms with J-aggregates. Opt. Lett. 2013, 38, 4498-4501.

(11) Torma, P.; Barnes, W. L. Strong coupling between surface plasmon polaritons and emitters: a review. Rep. Prog. Phys. 2015, 78, 78. 
(12) Iwase, H.; Englund, D.; Vuckovic, J. Analysis of the Purcell effect in photonic and plasmonic crystals with losses. Opt. Express 2010, 18, $16546-16560$.

(13) Koenderink, A. F. On the use of Purcell factors for plasmon antennas. Opt. Lett. 2010, 35, 4208-4210.

(14) Gonzalez-Tudela, A.; Huidobro, P. A.; Martin-Moreno, L.; Tejedor, C.; Garcia-Vidal, F. J.: Theory of Strong Coupling between Quantum Emitters and Propagating Surface Plasmons. Phys. Rev. Lett. 2013, 110.10.1103/PhysRevLett.110.126801

(15) Gomez, D. E.; Giessen, H.; Davis, T. J. Semiclassical Plexcitonics: Simple Approach for Designing Plexcitonic Nanostructures. J. Phys. Chem. C 2014, 118, 23963-23969.

(16) Balci, S.; Kocabas, C.; Ates, S.; Karademir, E.; Salihoglu, O.; Aydinli, A. Tuning surface plasmon-exciton coupling via thickness dependent plasmon damping. Phys. Rev. B: Condens. Matter Mater. Phys. 2012, 86.10.1103/PhysRevB.86.235402

(17) Nomura, M.; Kumagai, N.; Iwamoto, S.; Ota, Y.; Arakawa, Y. Laser oscillation in a strongly coupled single-quantum-dot-nanocavity system. Nat. Phys. 2010, 6, 279-283.

(18) Schwartz, T.; Hutchison, J. A.; Genet, C.; Ebbesen, T. W. Reversible Switching of Ultrastrong Light-Molecule Coupling. Phys. Rev. Lett. 2011, 106.10.1103/PhysRevLett.106.196405

(19) Zhou, N.; Yuan, M.; Gao, Y. H.; Li, D. S.; Yang, D. R. Silver Nanoshell Plasmonically Controlled Emission of Semiconductor Quantum Dots in the Strong Coupling Regime. ACS Nano 2016, 10, 4154-4163.

(20) Zengin, G.; Johansson, G.; Johansson, P.; Antosiewicz, T. J.; Kall, M.; Shegai, T. Approaching the strong coupling limit in single plasmonic nanorods interacting with J-aggregates. Sci. Rep. 2013, 3.10.1038/srep03074

(21) Zhang, Y. F.; Yang, D. J.; Wang, J. H.; Wang, Y. L.; Ding, S. J.; Zhou, L.; Hao, Z. H.; Wang, Q. Q. Multiple hybridized resonances of IR-806 chromonic molecules strongly coupled to $\mathrm{Au}$ nanorods. Nanoscale 2015, 7, 8503-8509.

(22) Simon, T.; Melnikau, D.; Sánchez-Iglesias, A.; Grzelczak, M.; Liz-Marzán, L. M.; Rakovich, Y.; Feldmann, J.; Urban, A. S. Exploring the Optical Nonlinearities of Plasmon-Exciton Hybrid Resonances in Coupled Colloidal Nanostructures. J. Phys. Chem. C 2016, 120, $12226-12233$.

(23) DeLacy, B. G.; Miller, O. D.; Hsu, C. W.; Zander, Z.; Lacey, S.; Yagloski, R.; Fountain, A. W.; Valdes, E.; Anquillare, E.; Soljacic, M.; Johnson, S. G.; Joannopoulos, J. D. Coherent Plasmon-Exciton Coupling in Silver Platelet-J-aggregate Nanocomposites. Nano Lett. 2015, 15, 2588-2593.

(24) Zengin, G.; Gschneidtner, T.; Verre, R.; Shao, L.; Antosiewicz, T. J.; Moth-Poulsen, K.; Käll, M.; Shegai, T. Evaluating Conditions for Strong Coupling between Nanoparticle Plasmons and Organic Dyes Using Scattering and Absorption Spectroscopy. J. Phys. Chem. C 2016, $120,20588$.

(25) Schlather, A. E.; Large, N.; Urban, A. S.; Nordlander, P.; Halas, N. J. Near-Field Mediated Plexcitonic Coupling and Giant Rabi Splitting in Individual Metallic Dimers. Nano Lett. 2013, 13, 32813286.

(26) Eizner, E.; Avayu, O.; Ditcovski, R.; Ellenbogen, T. Aluminum Nanoantenna Complexes for Strong Coupling between Excitons and Localized Surface Plasmons. Nano Lett. 2015, 15, 6215-6221.

(27) Wurthner, F.; Kaiser, T. E.; Saha-Möller, C. R. J-Aggregates: From Serendipitous Discovery to Supramolecular Engineering of Functional Dye Materials. Angew. Chem., Int. Ed. 2011, 50, 33763410.

(28) Zengin, G.; Wersall, M.; Nilsson, S.; Antosiewicz, T. J.; Kall, M.; Shegai, T. Realizing Strong Light-Matter Interactions between SingleNanoparticle Plasmons and Molecular Excitons at Ambient Conditions. Phys. Rev. Lett. 2015, 114.10.1103/PhysRevLett.114.157401

(29) Vasa, P.; Wang, W.; Pomraenke, R.; Lammers, M.; Maiuri, M.; Manzoni, C.; Cerullo, G.; Lienau, C. Real-time observation of ultrafast Rabi oscillations between excitons and plasmons in metal nanostructures with J-aggregates. Nat. Photonics 2013, 7, 128-132.
(30) Jin, R. C.; Cao, Y. W.; Mirkin, C. A.; Kelly, K. L.; Schatz, G. C.; Zheng, J. G. Photoinduced conversion of silver nanospheres to nanoprisms. Science 2001, 294, 1901-1903.

(31) Lee, P. C.; Meisel, D. Adsorption and Surface-Enhanced Raman of Dyes on Silver and Gold Sols. J. Phys. Chem. 1982, 86, 3391-3395.

(32) Nie, S. M.; Emery, S. R. Probing single molecules and single nanoparticles by surface-enhanced Raman scattering. Science 1997, $275,1102-1106$.

(33) Balci, S.; Kocabas, C.; Kucukoz, B.; Karatay, A.; Akhuseyin, E.; Yaglioglu, H. G.; Elmali, A. Probing ultrafast energy transfer between excitons and plasmons in the ultrastrong coupling regime. Appl. Phys. Lett. 2014, 105.05110510.1063/1.4892360

(34) Vanburgel, M.; Wiersma, D. A.; Duppen, K. The Dynamics of One-Dimensional Excitons in Liquids. J. Chem. Phys. 1995, 102, 2033.

(35) Hsu, C. W.; DeLacy, B. G.; Johnson, S. G.; Joannopoulos, J. D.; Soljacic, M. Theoretical Criteria for Scattering Dark States in Nanostructured Particles. Nano Lett. 2014, 14, 2783-2788.

(36) Vujacic, A.; Vasic, V.; Dramicanin, M.; Sovilj, S. P.; Bibic, N.; Hranisavljevic, J.; Wiederrecht, G. P. Kinetics of J-Aggregate Formation on the Surface of Au Nanoparticle Colloids. J. Phys. Chem. C 2012, 116, 4655-4661.

(37) Bradley, M. S.; Tischler, J. R.; Bulovic, V. Layer-by-layer Jaggregate thin films with a peak absorption constant of $10(6) \mathrm{cm}(-1)$. Adv. Mater. 2005, 17, 1881.

(38) Choi, Y. H.; Kang, T.; Lee, L. P. Plasmon Resonance Energy Transfer (PRET)-based Molecular Imaging of Cytochrome $\mathrm{c}$ in Living Cells. Nano Lett. 2009, 9, 85-90.

(39) Darby, B. L.; Auguie, B.; Meyer, M.; Pantoja, A. E.; Le Ru, E. C. Modified optical absorption of molecules on metallic nanoparticles at sub-monolayer coverage. Nat. Photonics 2016, 10, 40-U54.

(40) Wiederrecht, G. P.; Wurtz, G. A.; Bouhelier, A. Ultrafast hybrid plasmonics. Chem. Phys. Lett. 2008, 461, 171-179.

(41) Fofang, N. T.; Grady, N. K.; Fan, Z. Y.; Govorov, A. O.; Halas, N. J. Plexciton Dynamics: Exciton-Plasmon Coupling in a J-Aggregate$\mathrm{Au}$ Nanoshell Complex Provides a Mechanism for Nonllinearity. Nano Lett. 2011, 11, 1556-1560.

(42) Lidzey, D. G.; Fox, A. M.; Rahn, M. D.; Skolnick, M. S.; Agranovich, V. M.; Walker, S. Experimental study of light emission from strongly coupled organic semiconductor microcavities following nonresonant laser excitation. Phys. Rev. B: Condens. Matter Mater. Phys. 2002, 65.10.1103/PhysRevB.65.195312

(43) Agranovich, V. M.; Litinskaia, M.; Lidzey, D. G. Cavity polaritons in microcavities containing disordered organic semiconductors. Phys. Rev. B: Condens. Matter Mater. Phys. 2003, 67.10.1103/PhysRevB.67.085311

(44) Schwartz, T.; Hutchison, J. A.; Leonard, J.; Genet, C.; Haacke, S.; Ebbesen, T. W. Polariton Dynamics under Strong Light-Molecule Coupling. ChemPhysChem 2013, 14, 125-131.

(45) Zhong, X. L.; Chervy, T.; Wang, S. J.; George, J.; Thomas, A.; Hutchison, J. A.; Devaux, E.; Genet, C.; Ebbesen, T. W. Non-Radiative Energy Transfer Mediated by Hybrid Light-Matter States. Angew. Chem., Int. Ed. 2016, 55, 6202-6206. 